\title{
Rotational Dynamics of Anisotropic Microscopic Particles Studied by Second Harmonic Generation
}

\author{
Elsa C. Y. Yan and K. B. Eisenthal* \\ Department of Chemistry, Columbia Universiity, New York, New York 10027
}

Received: March 23, 2000; In Final Form: May 30, 2000

\begin{abstract}
This letter reports the application of second harmonic generation (SHG) to study the rotational dynamics of anisotropic microparticles. In this study, disk-shaped clay particles of montmorillonites with a diameter of $0.5 \mu \mathrm{m}$ and a thickness of $0.01 \mu \mathrm{m}$ suspended in liquid media were studied. Fluctuations in the SH signal observed upon irradiating the samples with the fundamental light are attributed to the rotational motion of the particles. The relaxation times obtained from the autocorrelation of the fluctuations are $0.024 \pm 0.04 \mathrm{~s}$ for water, $0.25 \pm 0.01$ for glycerol/water $(74: 26 \mathrm{w} / \mathrm{w})$, and $0.63 \pm 0.01 \mathrm{~s}$ for glycerol/water $(80: 20 \mathrm{w} / \mathrm{w})$. These values are of the same order of magnitudes for the relaxation times calculated using the StokesEinstein model for rotation of an oblate particle.
\end{abstract}

\section{Introduction}

Second harmonic generation (SHG) was applied to study the rotational dynamics of anisotropic microparticles. Since SHG from a microscopic centrosymmetric particle is the result of the coherent addition of the second harmonic fields from the particle surface, ${ }^{2}$ the SHG intensity depends on the shape as well as the orientation of the particle with respect to the incident and detected direction. Fluctuations in the SHG intensity were observed from anisotropic disk-shaped clay particles suspended in liquid media, which is attributed to the rotation of the anisotropic particles in the liquid media. These fluctuations were not observed for the spherical microscopic particles: polystyrene beads and oil droplets in an oil/water emulsion. The rotational relaxation time of anisotropic particles can be obtained from an analysis of the fluctuations. In these studies, disk-shaped clay particles are montmorillonites with a diameter of $0.5 \mu \mathrm{m}$ and a thickness of $0.01 \mu \mathrm{m}$ and were suspended in water or in a mixture of glycerol and water. The size distribution of the clay particles is $0.5 \pm 0.15 \mu \mathrm{m}$. We have found that the relaxation time depends on the viscosity of the media, $0.024 \pm 0.04 \mathrm{~s}$ for water, $0.25 \pm 0.01$ for glycerol/water $(76: 24 \mathrm{w} / \mathrm{w})$, and $0.63 \pm$ $0.01 \mathrm{~s}$ for glycerol/water $(80: 20 \mathrm{w} / \mathrm{w})$. These values are consistent with the rotational relaxation times calculated by approximating the clay particles by an oblate structure of the same dimensions.

Surface specific second harmonic generation (SHG) is a second-order spectroscopic technique, which involves conversion of incident light at a fundamental frequency $\omega$ to light at twice the frequency $2 \omega$ via a nonlinear interaction with the medium. ${ }^{1}$ Under the dipole approximation, SHG is forbidden in centrosymmetric media, e.g., in bulk solution. Since surfaces are intrinsically noncentrosymmetric, SHG becomes dipoleallowed, and thus is surface specific. This surface selectivity has made SHG a powerful method for probing dynamic and equilibrium processes at interfaces. ${ }^{1}$

SHG has recently been shown to be a new way to study microparticle surfaces. ${ }^{2}$ Since the local regions of the surfaces of microparticles can be noncentrosymmetric even when the particle, e.g., a sphere, is centrosymmetric, the second harmonic field can be generated at the surface of the particle upon irradiation with fundamental light. If the size of the microparticle is comparable to the coherence length for SHG, approximately wavelength of the light, the second harmonic field generated locally can add constructively, yielding a SHG signal. Taking advantage of both the surface specificity and spectroscopic selectivity of SHG, we have applied SHG to study various micron- and submicron-sized particles, which include the aqueous suspensions of polystyrene beads, ${ }^{2,3}$ liposomes ${ }^{4}$ semiconductor particles, ${ }^{5}$ oil/water emulsions, ${ }^{5}$ and disk-shaped clay particles of montmorillonites. ${ }^{6}$

The translational and rotational motions of long chain molecules at the planar air/water interface have been studied also by SHG measurements. ${ }^{7-9}$ SHG is sensitive to both the interface molecular density and molecular orientation. As the molecules rotate and diffuse at the interface, fluctuations in the SHG were observed. A translational diffusion constant for the motion of interfacial clusters was found, ${ }^{9}$ and a new orientational phase transition of a monolayer at the air-water interface was observed. ${ }^{7,8}$

The anisotropic particles used in the present study are diskshaped montmorillonite particles. A montmorillonite particle is centrosymmetric, and therefore does not give an SHG signal from the bulk. It is composed of about 10 unit layers, and each layer consists of an octahedral sheet of alumina sandwiched between two tetrahedral sheets of silica. ${ }^{10}$ The basal surfaces of the particles are negatively charged due to isomorphous substitution of aluminum or silicon by other metal ions of lower valence. The edge surfaces are positively charged in neutral or acidic environment due to the broken and hydrolyzed $\mathrm{Al}-\mathrm{O}$ and $\mathrm{Si}-\mathrm{O}$ bonds. Since the static electric field due to the surface charges extends into the bulk solution and polarizes the solvent molecules, the centrosymmetry of the bulk solution near the charged surface is broken, which leads to the generation of a SHG signal. ${ }^{11}$

In a previous study, we reported on the SHG signal detected from montmorillonite particles. ${ }^{6}$ We observed a SHG signal from organic molecules, 4-(3-pyridylazo)resorcinol (PR) adsorbed on the surface of the clay particles, and we also observed the SHG signal from the clay particles in the absence of the organic molecule, PR. In the absence of the organic molecule PR, the SHG signal is attributed to the broken centrosymmetry of the bulk water molecules polarized by the surface charges on the clay surface in the edge region. However, a closer analysis reveals that the contribution of SHG from the edge 
plane and basal planes of a disk-shaped particle can be comparable. The reason is that the area of the basal planes is much larger than that of the edge plane. The second harmonic field scales with the surface area and with a size parameter due to interference from opposing surfaces given by a separation length. The size parameter is some dimension of the particle divided by the wavelength of the light, $\lambda$. The second harmonic fields contributed from edge plane and basal plane are as follows.

edge plane:

$$
E_{2 \omega} \sim \text { size parameter } \times \text { area } \sim(r / \lambda)(r d)=r^{2} d / \lambda
$$

basal plane:

$$
E_{2 \omega} \sim \text { size parameter } \times \text { area } \sim(d / \lambda)\left(r^{2}\right)=r^{2} d / \lambda
$$

where $r$ is the radius and $d$ is the thickness of the disk. Therefore, the SHG signal observed from the aqueous suspension of clay particles can result from both edge and basal surfaces. It is to be noted that both contributions depend on the orientation of the disk-shaped particle with respect to the incident and detection directions.

As noted earlier, fluctuations in the SHG signal observed from clay particles were not observed from isotropic particles that we have studied, e.g., polymer microspheres, liposomes, oil/ water emulsions, and semiconductor particles. We also found that the fluctuation of the SHG signal from the clay particles depends on the viscosity of the media. Analyzing the fluctuation by an intensity correlation function, the description yields the relaxation time of the clay particle motion. The obtained values are compared with theoretical rotational and translational relaxation times. Our experimental values are consistent with the theoretical rotational relaxation time and not with translational motion in to and out of the incident beam volume.

Depolarized dynamic light scattering has been used to study the rotational dynamics of optically anisotropic particles of submicron size. ${ }^{12}$ In the depolarized dynamic light scattering method, the fluctuation of the intensity of the depolarized linearly scattered light is measured, while in the SHG method, the fluctuation of the intensity of SHG is measured. The fluctuation of the SHG intensity is background free because the input fundamental light is at frequency $\omega$ and the detected SHG is at frequency $2 \omega$.

\section{Background Theory}

In general, a second-order optical process can be described as ${ }^{1,13}$

$$
\vec{E}_{2 \omega}=\alpha^{(2)}: \vec{E}_{\omega} \vec{E}_{\omega}
$$

where $\vec{E}_{2 \omega}$ is the second harmonic field, $\vec{E}_{\omega}$ is the fundamental field, and $\alpha^{(2)}$ is the second-order polarizability, which is a third rank tensor. The second harmonic signal observed from a microparticle is the result of the coherent addition of second harmonic field generated from the surface of the particle, which depends on the size of the particle. ${ }^{2}$ When the particle is much smaller than the wavelength of the light, the coherent SHG signal will be greatly reduced. Recently, the theory of SHG generated from the surfaces of particles that are small compared to the wavelength of light has been developed. ${ }^{14}$ However, a complete theory describing the second harmonic process at microparticle surfaces has not yet been established. To simplify the discussion, an anisotropic particle is considered to have an effective anisotropic second-order polarizability, $\alpha^{(2)}$. The

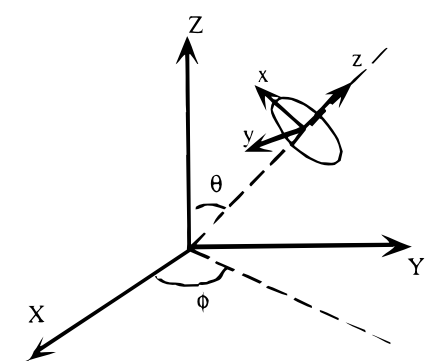

Figure 1. Definition of coordinates. Euler angles between the laboratory coordinates $(X, Y, Z)$ and the molecular coordinates $(x, y$, z).

problem is now reduced to the one similar to linear optical processes of dynamic light scattering from cylindrically symmetric molecules ${ }^{15}$ and fluorescence depolarization by anisotropic rotational diffusion, ${ }^{16}$ which have already been solved and discussed in detail. ${ }^{14,15}$ In the following discussion, we consider a laboratory-fixed polarizability tensor with the projection of molecular-fixed polarizability tensor onto the laboratory axes.

We choose the coordinate system as shown in Figure 1. Let a set of Cartesian coordinates $(x, y, z)$ be the three principle diffusion axes fixed in the disk and $(X, Y, Z)$ be the laboratory fixed axes. Because rotations about the $z$ axis of the disk do not change the second-order polarizability in the laboratory frame, such rotations do not contribute to the observed fluctuations in the SHG signal. Since only the rotations of the disk about the $x$ or $y$ axes give rise to SHG fluctuations, and furthermore the rotational friction about the $x$ and $y$ axes is the same by symmetry, there will be only one rotational relaxation time, i.e., a single rotational diffusion coefficient, $D$.

In the laboratory frame, the second harmonic field polarized along the $i$ axis can be expressed as

$$
E_{2 \omega}^{i}(\Omega, t)=\sum_{j k} \alpha_{i j k}^{(2)}(\Omega, t) E_{\omega}^{j} E_{\omega}^{k}
$$

where $E_{\omega}^{j, k}$ is the incident fundamental fields polarized along the $j$ or $k$ axes in the laboratory frame respectively, and $\alpha_{i j k}^{(2)}$ is one of the 27 elements of the laboratory fixed second-order polarizability tensor, $\alpha^{(2)}$. This formula contains the projection of the molecular-fixed polarizability tensor onto the incident and detected polarization directions of the light wave. As the molecule rotates, the projections of the tensor onto laboratory axes $(X, Y, Z)$ change. Therefore, its component $\alpha_{i j k}^{(2)}(\Omega, t)$ is a function of time $(t)$ as well as the orientation $(\Omega)$ of the particle.

To further simplify the discussion, we use a vertically polarized fundamental field and detect a vertically polarized second harmonic field, i.e., $i=j=k=Z$. The second harmonic intensity generated from one particle can be written as

$$
I_{\mathrm{VV}}(\Omega, t)=\left|E_{2 \omega}^{Z}(\Omega, t)\right|^{2}=\left|\alpha_{\mathrm{ZZZ}}^{(2)}(\Omega, t) E_{\omega}^{Z} E_{\omega}^{Z}\right|^{2}
$$

The correlation function, $C(t)$, of the detected second harmonic intensity from an irradiated volume of particles can then be written as

$$
\begin{aligned}
& \left\langle I_{\mathrm{VV}}\left(\Omega_{0}, 0\right) I_{\mathrm{VV}}(\Omega, t)\right\rangle= \\
& \quad \int \mathrm{d} \Omega_{0} \int \mathrm{d} \Omega I_{\mathrm{VV}}\left(\Omega_{0}, 0\right) G\left(\Omega, t ; \Omega_{0}, 0\right) I_{\mathrm{VV}}(\Omega, t)
\end{aligned}
$$

where $G\left(\Omega, t ; \Omega_{0}, 0\right)$ is the joint probability density of finding a disk with orientation $\Omega_{0}$ initially and at $\Omega$ at time $t$. $G\left(\Omega, t \mid \Omega_{0}, 0\right)$, which is the solution of the rotational diffusion equation, is 


\section{SCHEME 1}

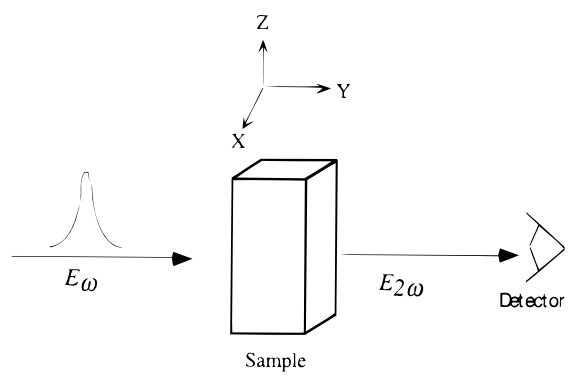

expressed in terms of spherical harmonics, $Y_{l, m}(\theta, \phi)$ and as discussed earlier a single rotational diffusion constant, $D$, for the oblate shaped clay particle. ${ }^{14,15}$

When the incident fundamental wave is vertically polarized, the particle system remains symmetrical with respect to $Z$ and $G\left(\Omega, t \mid \Omega_{0}, 0\right)$ is independent of $\phi$. Substituting the expansion of $G\left(\Omega, t \mid \Omega_{0}, 0\right)$ in terms of $Y_{l, m}(\theta, \phi)$ and $D$, factoring out the integration over initial orientation as a constant, and arbitrarily choosing $\theta=\phi=0$, we can write eq 4 as

$$
\begin{aligned}
&\left\langle I_{\mathrm{VV}}\left(\Omega_{0}, 0\right) I_{\mathrm{VV}}(\Omega, t)\right\rangle \sim \sum_{l} \frac{(2 l+1)}{4 \pi} \times \\
& \quad \int_{0}^{\pi} \int_{0}^{2 \pi} \sin \theta \mathrm{d} \theta \mathrm{d} \phi\left|\alpha_{\mathrm{ZZZ}}^{(2)}(\theta, \phi, t)\right|^{2} P_{l}(\cos \theta) \mathrm{e}^{-l(l+1) D t}
\end{aligned}
$$

Since $\alpha_{\mathrm{ZZZ}}^{(2)}(\theta, \phi, t)$ is the element of a third rank tensor, $\left|\alpha_{\mathrm{ZZZ}}^{(2)}(\theta, \phi, t)\right|^{2}$ is a trigonometric function, e.g., $\cos \theta$, to the sixth power. Because of the characteristics of the Legendre polynomial, $P_{l}(\cos \theta)$, it follows that the integrals in the summation in eq 5 do not vanish for $l=0,2,4$, and 6 . Therefore, the correlation function can be written as

$$
C(t) \sim k_{l=0}+k_{l=2} \mathrm{e}^{-6 D t}+k_{l=4} \mathrm{e}^{-20 D t}+k_{l=6} \mathrm{e}^{-42 D t}
$$

where $k_{l=0,2,4,6}$ is the preexponential factors for the corresponding $l$.

\section{Experimental Section}

The setup for the second harmonic measurement is described in detail elsewhere. ${ }^{3}$ Briefly, it consists of a Ti:sapphire oscillator, which provides $100 \mathrm{fs}$ pulses at $844 \mathrm{~nm}$ at a repetition rate of $82 \mathrm{MHz}$. The fundamental light was focused into a sample cell by an $f=7.5 \mathrm{~cm}$ lens giving a spot size of $30 \mu \mathrm{m}$. Filters and a monochromator were used to separate the SHG output signal from any background signal. Both the incident fundamental and the detected second harmonic light are vertically polarized. The fluctuation of the $\mathrm{SH}$ signal is monitored by a single photon counting system, Scheme 1 .

All aqueous solutions were prepared in doubly distilled water. The clay sample of $\mathrm{Na}$-montmorillonite was stored in $0.5 \mathrm{M}$ $\mathrm{NaCl}$ solution until used. The suspension was centrifuged (15 $000 \mathrm{rpm}$ for $5 \mathrm{~min}$ ), resuspended, and washed with doubly distilled deionized water until a negative chloride test was obtained with silver nitrate. The concentration of washed clay particles in aqueous solution was determined by a simple weighing method. The experiments were conducted at room temperature, $22^{\circ} \mathrm{C}$.

\section{Results and Discussion}

The time dependence of SHG signals are shown in Figure 2 for the clay particles suspended in the three solvents: (a) water, (b) glycerol/water (76:24 w/w), and (c) glycerol/water (80:20
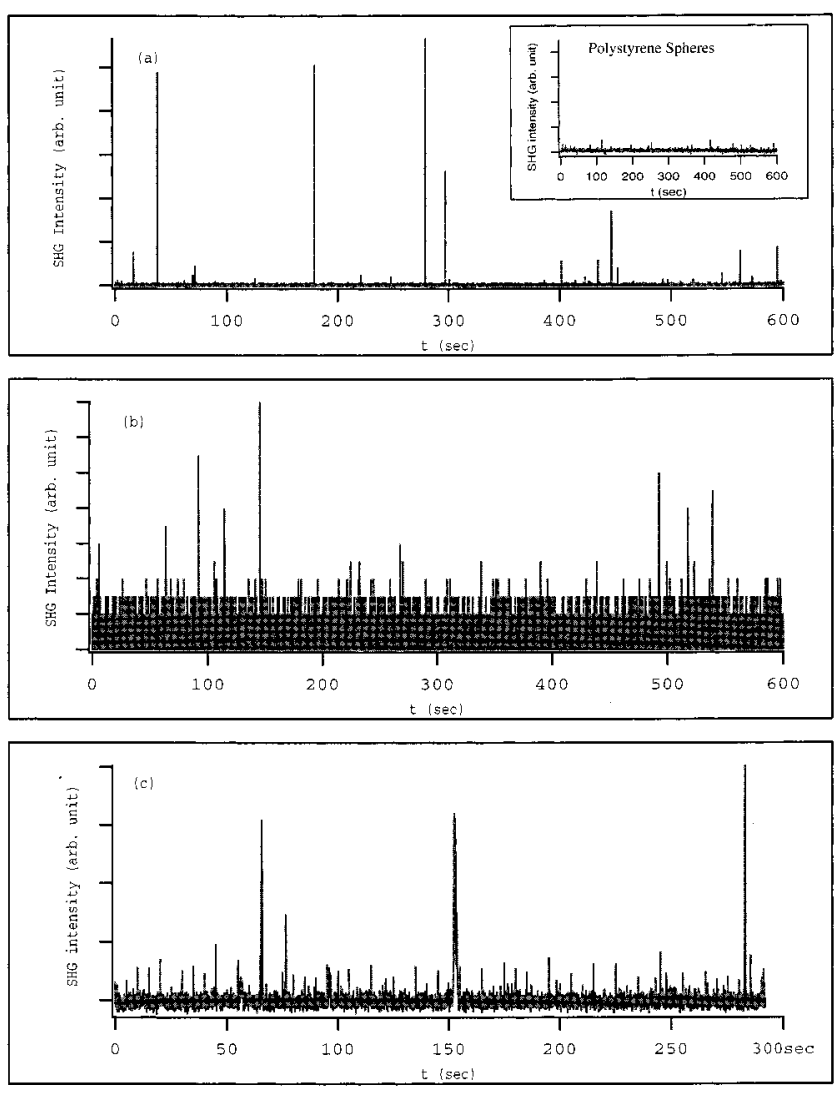

Figure 2. SHG intensity monitored as a function of time. (a) Clay particles in water at particle density $7 \times 10^{6}$ particles $/ \mathrm{cm}^{3}$ with time resolution $0.0059 \mathrm{~s}$. (b) Clay particles in glycerol/water $(76: 24 \mathrm{w} / \mathrm{w})$ at particle density $7 \times 10^{7}$ particles $/ \mathrm{cm}^{3}$ with time resolution $0.0058 \mathrm{~s}$. (c) Clay particles in glycerol/water $(80: 20 \mathrm{w} / \mathrm{w})$ at particle density 1 $\times 10^{10}$ particles $/ \mathrm{cm}^{3}$ with time resolution $0.037 \mathrm{~s}$. The inset in (a) is the time dependence of the SHG signal from a suspension of spherical polystyrene particles at a density $3 \times 10^{10}$ particles $/ \mathrm{cm}^{3}$. The normalized average signal is $1 \pm 0.016$.

w/w) and at different particle densities as indicated. The time dependence of the SHG signal for spherical polystyrene particle of $0.24 \pm 0.01 \mu \mathrm{m}$ suspended in water is shown in the inset in Figure 1a. The deviation shows that the fluctuations of the SHG signal observed from disk-shaped clay particles are not observed from the spherical polystyrene particles. The fluctuations observed from the clay samples were not observed from neat solvents.

The fluctuations analyzed by autocorrelation functions, are shown corresponding to Figure $3 a-c$. They are fitted to a singleexponential function, shown as solid lines in Figure 3. They are also fitted into a double-exponential function resulting in two very similar time constants without significant improvement of the fitting. The deviation of the data from the singleexponential fit may be due to the size distribution of the clay sample, $0.5 \pm 0.15 \mu \mathrm{m}$. The measurements and analysis were repeated four times for clay particles suspended in water, yielding an average decay time of $0.024 \pm 0.04 \mathrm{~s}$, and repeated twice for clay particles in glycerol/water $(74: 26 \mathrm{w} / \mathrm{w})$ and glycerol/water (80:20 w/w), yielding decay times of $0.25 \pm 0.01$ and $0.63 \pm 0.01 \mathrm{~s}$, respectively. These experimental results agree with the fact that the higher the viscosity the longer the relaxation time.

The rotational relaxation times obtained by the SHG measurement are compared with the theoretical values calculated by approximating the disk-shaped clay particles to an oblate ellipsoid with the axial ratio equal to the ratio of the diameter 

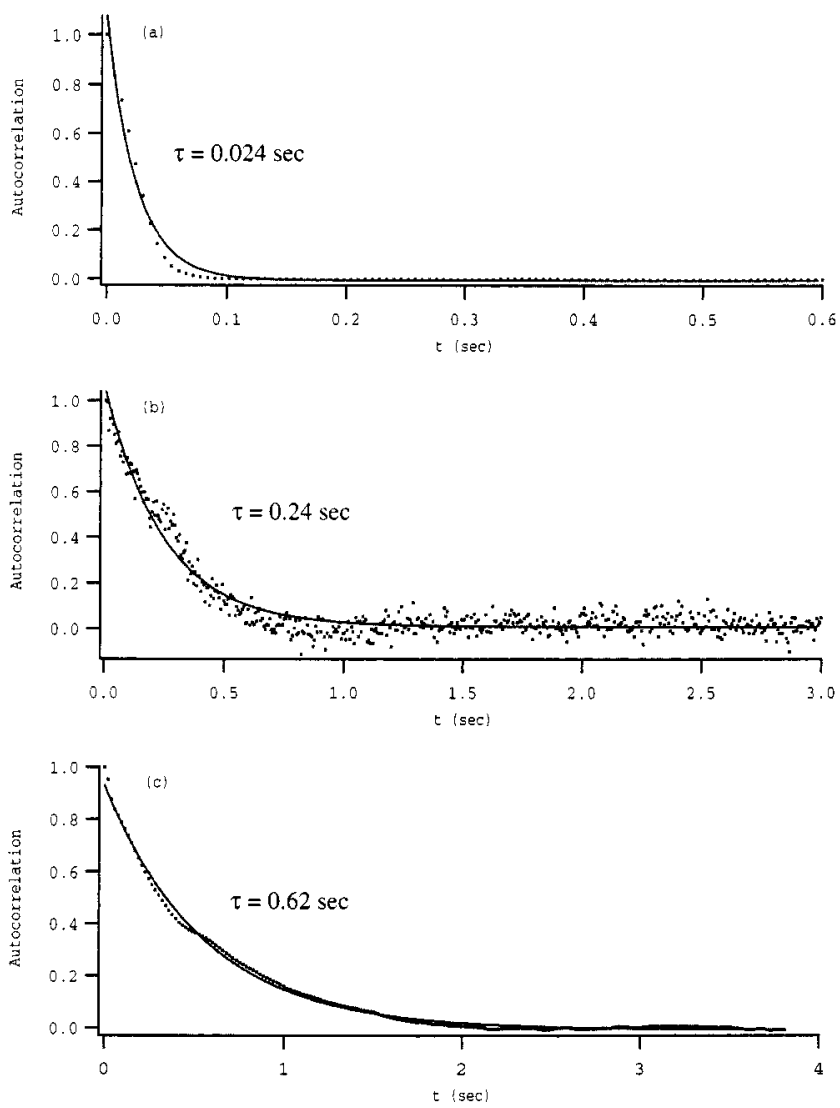

Figure 3. Autocorrelation of the SHG fluctuation in Figure 2. (a) Clay particles in water. (b) Clay particles in glycerol/water (74:26 w/w). (c) Clay particles in glycerol/water $(80: 20 \mathrm{w} / \mathrm{w})$. The solid lines are the exponential fits.

to the thickness of the clay particle and of the same volume in various solvents. We used the expression derived by Perrin in $1934,{ }^{17,18}$ to calculate the rotational relaxation time of the ellipsoid. The calculated rotational relaxation time of the oblate about the long axis are found to be $0.010 \mathrm{~s}$ in water. In the calculation, we used the slowest decay that is $t=1 / 6 \mathrm{D}$ in eq 6 because the faster ones are unobservable using our experimental setup having a time resolution of $6 \mathrm{~ms}$. The viscosity of glycerol/ water (76:24 w/w) and glycerol/water (80:20 w/w) are 27 times and 60 times that of water, thus yielding the calculated rotational relaxation times of $0.27 \mathrm{~s}$ for glycerol/water $(76: 24 \mathrm{w} / \mathrm{w})$ and $0.60 \mathrm{~s}$ for glycerol/water $(80: 20 \mathrm{w} / \mathrm{w})$.

The time scale of the observed relaxation shows that the fluctuation of the SHG signal is not correlated to the translational motion of the particles. The translational constant of the diskshaped particle in water is calculated ${ }^{17}$ by approximating the disk to an oblate ellipsoid with the dimension of the clay particle and found to be $1.2 \times 10^{-12} \mathrm{~m}^{2} \mathrm{~s}^{-1}$. Using the EinsteinSmoluchowski equation, $\left\langle(\Delta r)^{2}\right\rangle=4 D \Delta t$, where $\Delta r$ is the net displacement in the radial direction that occurs in time $\Delta t$ and $D$ is the translational diffusion constant, we estimate the translational relaxation time $\Delta t$. The spot size of our laser beam has a diameter of $30 \mu \mathrm{m}$. We substitute $\Delta r=15 \mu \mathrm{m}$, and $D=$ $1.2 \times 10^{-12} \mathrm{~m}^{2} \mathrm{~s}^{-1}$, we find that $\Delta t$ is $46 \mathrm{~s}$. Our measured relaxation time is of the order of $10^{-2} \mathrm{~s}$.

We have shown that the measured relaxation times are of the same order of magnitude as the calculated ones. Because of the broad size distribution of the clay particles, $0.5 \pm 0.15$, some deviation between the theoretical and experimental values are expected. It is calculated that for a $30 \%$ increase in the diameter of the clay particle the calculated rotational relaxation time would double. The reason for the measured results deviating differently from the calculated ones for pure water and water/glycerol mixtures is attributed to the different extent of swelling of the clay particles in pure water and the binary solvent of water/glycerol. It is known that in binary solvent systems, a mixture of water and an organic solvent, the swelling processes of montmorillonite particles is very sensitive to the composition of the solvent. Changing the composition of the solvent could make the clay particles expand to different thicknesses, ${ }^{19}$ resulting in a different rotational relaxation time. That can also explain the observation that the measured rotational times do not scale exactly linearly with the viscosity of the media.

\section{Conclusions}

We demonstrate that second harmonic generation can be applied to investigate the rotational dynamics of anisotropic microparticles. The rotational relaxation times of the disk-shaped clay particles of montmorillonites with a diameter of $0.5 \mu \mathrm{m}$ and a thickness of $0.01 \mu \mathrm{m}$ was found to be $0.024 \pm 0.04 \mathrm{~s}$ for water, $0.25 \pm 0.01 \mathrm{~s}$ for glycerol/water $(74: 26 \mathrm{w} / \mathrm{w})$, and 0.63 $\pm 0.01 \mathrm{~s}$ for glycerol/water $(80: 20 \mathrm{w} / \mathrm{w})$ at $22{ }^{\circ} \mathrm{C}$, which is consistent with the Stokes-Einstein calculation of rotational relaxation time for an ellipsoid approximating the dimension of the clay particles.

Acknowledgment. We thank Prof. C. Johnston (Prudue) for providing us the clay sample of $\mathrm{Na}$-montmorillonite. The contribution from Dr. J. I. Dadap, Dr. D. Zimdars, and Dr. X. Zhao to this work is gratefully acknowledged. We thank the National Science Foundation for their support.

\section{References and Notes}

(1) Shen, Y. R. Annu. Rev. Phys. Chem. 1989, 40, 327. Eisenthal, K. B. Annu. Rev. Phys. Chem. 1992, 43, 627. Eisenthal, K. B. Chem. Rev. 1996, 96, 1343. Corn, R. M.; Higgins, D. A. Chem. Rev. 1994, 94, 107. Eisenthal, K. B. J. Phys. Chem. 1996, 100, 12997.

(2) Wang, H.; Yan, E. C. Y.; Borguet, E.; Eisenthal, K. B. Chem. Phys. Lett. 1996, 259, 1343.

(3) Wang, H.; Yan, E. C. Y, Liu, Y.; Eisenthal, K. B J. Phys. Chem. B 1998, 102, 4446. Yan, E. C. Y, Liu, Y.; Eisenthal, K. B. J. Phys. Chem. $B$ 1998, 102, 6331 .

(4) Yan, E. C. Y.; Eisenthal, K. B. Biophys. J., in press. Sribastava, A.; Eisenthal, K. B. Chem. Phys. Lett. 1998, 292, 345.

(5) Liu, Y.; Dadap, J. I.; Zimdars, D.; Eisenthal, K. B. J. Phys. Chem. B 1999, 103, 2480 .

(6) Yan, E. C. Y.; Eisenthal, K. B. J. Phys. Chem. B 1999, 103, 6056.

(7) Zhao, X.; Subrahmanyan, S.; Eisenthal, K. B. Phys. Rev. Lett. 1991, 67, 2025 .

(8) Zhao, X.; Eisenthal K. B. J. Chem. Phys. 1995, 102, 5818.

(9) Zhao, X.; Goh, C.; Subrahmanyan, S.; Eisenthal, K. B. J. Phys. Chem. 1990, 94, 3370.

(10) VanOlphen, H. An Introduction to Clay Colloid Chemistry; Wiley: New York, 1963.

(11) Ong, S.; Zhao, X.; Eisenthal, K. B. Chem. Phys. Lett. 1992, 191, 327. Zhao, X.; Ong, S.; Eisenthal, K. B. Chem. Phys. Lett. 1993, 202, 513. Zhao, X.; Ong, S.; Wang, H.; Eisenthal, K. B. Chem. Phys. Lett. 1993, 214, 203.

(12) Hsu, P.; Poulin, P.; Weitz, D. A. J. Colloid Interface Sci. 1998, 200, 182. Piazza, R.; Degiorgio, V. Phys. Rev. Lett. 1991, 67, 27. Piazza, R.; Stavans, J.; Bellini, T.; Degiorgio. V. Opt. Commun. 1989, 73, 4.

(13) Shen, Y. R. The Principle of Nonlinear Optics; Wiley: New York, 1984.

(14) Dadap, J. I.; Shan, J.; Eisenthal, K. B.; Heinz, T. F. Phys. Rev. Lett. 1999, 83, 4045 .

(15) Berne, B. J.; Pecora, R. Dynamic Light Scattering; John Wiley \& Son: New York, 1976; p 121.

(16) Chuang, T. J.; Eisenthal, K. B. J. Chem. Phys. 1972, 57, 5094

(17) Perrin, F. J. Phys. Rad. Ser. 1934, 5, 497.

(18) Cantor, C. R.; Schimmel, P. R. Biophysical Chemistry; W. H. Freeman: San Francisco, 1980; Vol. 2, p 560. 727. 